\title{
Evolution of the ABO blood group gene in Japanese macaque
}

\author{
Reiko Noda ${ }^{1,2}$, Takashi Kitano ${ }^{1,4}$, Osamu Takenaka ${ }^{3}$, and Naruya Saitou ${ }^{1,2, *}$ \\ ${ }^{1}$ Laboratory of Evolutionary Genetics, National Institute of Genetics, Mishima 411-8540, Japan \\ ${ }^{2}$ Department of Genetics, School of Life Science, The Graduate University for Advanced \\ Studies, Mishima 411-8540, Japan \\ ${ }^{3}$ Primate Research Institute, Kyoto University, Inuyama 484-8506, Japan \\ ${ }^{4}$ Present address: Max-Planck Institute of Molecular Anthropology, \\ Leipzig, Germany
}

(Received 18 December, 1999, accepted 31 May 2000)

\begin{abstract}
We determined 5 sequences of Japanese macaque ABO blood group gene exon 7 (ca. $0.5 \mathrm{~kb}$ ) and 2 sequences for exon 5 and intron 6 (ca. $1.7 \mathrm{~kb}$ ). We compared those data with published sequences of other Old World monkey species, and the results suggest that alleles A and B were polymorphic in the ancestral species of macaques, and that $\mathrm{B}$ type allele evolved independently in macaque and baboon lineages.
\end{abstract}

\section{INTRODUCTION}

The human ABO blood group was discovered by Landsteiner, and its mode of inheritance as multiple alleles at a single genetic locus was established by Bernstein (see Crow [1993] for review). Immunodominant ABH antigens are carbohydrate structures of glycoproteins and glycolipids (see Yamamoto [1995] for review). ABO alleles A and B thus code for glycosyltransferases which transfer GalNAc and galactose, respectively, while $\mathrm{O}$ is a null allele incapable of coding for a functional glycosyltransferase. Yamamoto et al. (1990) determined the cDNA sequences of three common alleles, $\mathrm{A} 1, \mathrm{~B}$, and $\mathrm{O}$, and Yamamoto et al. (1995) determined the genomic organization of the gene.

Existence of A-like and B-like antigens in non-human primates were known (reviewed in Blancher and Socha [1997]). Serological typing of variety of primate species revealed the presence of an A-like antigen in common chimpanzee (Pan troglodytes: $90 \%$ of the typed individuals), bonobo or pygmy chimpanzee (Pan paniscus: 100\%), gibbon (Hylobates: 19\%), orangutan (Pongo pygmaeus: $56 \%$ ), and most Old World monkey species $(0-100 \%)$ as well as some New World monkey species (22-100\%). Similarly, a B-like antigen was detected in all the gorilla subspecies (Gorilla gorilla gorilla, G. g. graueri, G. g. beringei: $100 \%$ ), gibbon (42\%), and virtually all Old World monkey species (0-100\%) as well as some New World monkey species (27-100\%; see Blancher and Socha [1997] for review). These serological typing data suggested that A-like and B-like antigens arose early in evolution of pri-

Edited by Naoyuki Takahata

* Corresponding author. E-mail: nsaitou@genes.nig.ac.jp mates, possibly before the divergence of the Platyrrhini and Catarrhini. This may be called "trans-specific polymorphism" hypothesis, and was first proposed by Bernard and Ruffié (1972).

Kominato et al. (1992) determined the $\mathrm{ABO}$ group gene exon 7 sequences of chimpanzee, gorilla, orangutan, crabeating macaque, and yellow baboon. Martinko et al. (1993) supported the trans-species polymorphism hypothesis in hominoids based on their sequence data. Saitou and Yamamoto (1997) analyzed sequence data of the ABO blood group gene exon 7 that determines the activity of the glycosyltransferase-encoding genes from the human, chimpanzee, gorilla, orangutan, and some Old World monkeys. They proposed that the common ancestral gene for the hominoid and Old World monkey ABO blood group gene was A type, and that $\mathrm{B}$ alleles evolved independently in human, gorilla, and baboon lineages. O'hUigin et al. (1997) determined the ABO blood group gene partial sequences between exon 5 and exon 7 of human, chimpanzee, and gorilla. They also suggested that the $\mathrm{B}$ allele evolved independently in human and gorilla.

Diamand et al. (1997) determined the A, B, and O allele nucleotide sequences of olive baboon. Doxiadis et al. (1998) determined sequences of human, rhesus macaque, and crab-eating macaque for $\mathrm{ABO}$ blood group genes exon 7 and they showed that all $O$ type alleles so far found in primates were considered to be inactivated $\mathrm{A}$ genes, and that $\mathrm{A}$ and $\mathrm{B}$ reactivity was generated independently in the hominoid and Old World monkey lineages. Kermarrec et al. (1999) determined sequences of O type alleles for two rhesus macaque and one chimpanzee. They also suggested that A and B reactivities were generated independently in hominoid and Old World monkey lineages, and that $\mathrm{O}$ alleles are species-specific, resulting 
from independent silencing mutations. Those observations reinforce the hypothesis that the polymorphism in primates reflects convergent evolution rather than transpecies inheritance of ancestral alleles.

Japanese macaque is phylogenetically closely related species of crab-eating macaque and rhesus macaque (e.g., Hayasaka et al., 1996), and there is only B type in Japanese macaque (Blancher and Socha, 1997). The purpose of this paper is to determine and to analyze the sequence of Japanese macaque ABO blood group genes. Evolution of ABO blood group gene in Old World monkey will be discussed based on the novel sequence data.

\section{MATERIALS AND METHOD}

The genomic DNA samples for five Japanese macaque (Macaca fuscata) individuals collected by one of us (O.T.) were used.

PCR was carried out using specific primers based on human and chimpanzee exon 7, and based on chimpanzee and gibbon exon 6. Primers are: SN-10, an exon 7 down- stream specific primer, 5'-CGATGCCGTTGGCCTGGTC3', SN-02, an exon 7 upstream specific primer, 5'-GAGACGGCGGAGAAGCACTT-3', and SN-15, an exon 6 specific primer, 5'-AGCAGTTCAGGCTCCAGAACAC3'.

Each PCR reaction mixture contained $160 \mathrm{mM}$ each of dATP, dTTP, dCTP and dGTP, $150 \mu \mathrm{M} \mathrm{MgCl}_{2}, 1 \times$ reaction buffer containing no $\mathrm{Mg}^{2+}$ ion, $1 \mathrm{mM}$ of each primer, and 2 unit Taq polymerase (TOYOBO and NIPPON GENE). About 200ng of genomic DNA was used as template. The PCR program consisted of 20-45 cycles of 1-min denaturation at $94^{\circ} \mathrm{C}$ followed by 1 -min primer annealing at $65^{\circ} \mathrm{C}$ or $68^{\circ} \mathrm{C}$ and 2 -min extension at $72^{\circ} \mathrm{C}$. Immediately preceding and following these $20-45$ cycles, a 9-min hotstart step at $94^{\circ} \mathrm{C}$ and 60 -min extension step at $72^{\circ} \mathrm{C}$ were included, respectively. We used GeneAmp PCR system 2400 and 9700 (PE Biosystems).

PCR products were confirmed by $1.5 \%$ agarose gel eletctrophoresis, and purified using Micro Spin Columns (Pharmacia Biotech). The purified PCR products were sequenced by the dideoxy chain-termination method using fluorescent ddNTPs, sequenase enzyme and buffer

Table 1. Nucleotide sequences of the Old World monkey ABO blood group genes used in this study

\begin{tabular}{|c|c|c|c|c|}
\hline $\begin{array}{l}\text { Sequence } \\
\text { name }\end{array}$ & $\begin{array}{r}\text { Allele } \\
\text { type }\end{array}$ & $\begin{array}{l}\text { Length } \\
\text { (bp) }\end{array}$ & Reference & $\begin{array}{l}\text { Accession } \\
\text { number }\end{array}$ \\
\hline \multicolumn{5}{|c|}{ Japanese macaque } \\
\hline JaM-B1 & $\mathrm{B}$ & 487 & This study (sample ID \#011) & AB041525 \\
\hline JaM-B2 & $\mathrm{B}$ & 487 & This study (sample ID \#012) & AB041526 \\
\hline JaM-B3 & $\mathrm{B}$ & 487 & This study (sample ID \#013) & AB041527 \\
\hline JaM-B4 & $\mathrm{B}$ & $1734^{\mathrm{a}}$ & This study (sample ID \#014) & AB041528 \\
\hline JaM-B5 & $\mathrm{B}$ & $1678^{\mathrm{b}}$ & This study (sample ID \#015) & AB041529 \\
\hline \multicolumn{5}{|c|}{ Crab eating macaque } \\
\hline CeM-A1 & $\mathrm{A}$ & 571 & Macaque of Kominato et al. (1992) & - \\
\hline CeM-O & $\mathrm{O}$ & 559 & Cy*O101 of Doxiadis et al. (1998) & AF052081 \\
\hline CeM-A2 & $\mathrm{A}$ & 471 & Cy*A102 of Doxiadis et al. (1998) & AF052078 \\
\hline CeM-A3 & $\mathrm{A}$ & 571 & Cy*A103 of Doxiadis et al. (1998) & AF052079 \\
\hline CeM-B1 & $\mathrm{B}$ & 571 & Cy*B101 of Doxiadis et al. (1998) & AF052082 \\
\hline CeM-B2 & $\mathrm{B}$ & 571 & Cy*B102 of Doxiadis et al. (1998) & AF052083 \\
\hline \multicolumn{5}{|c|}{ Rhesus macaque } \\
\hline RhM-A1 & $\mathrm{A}$ & 571 & Rh*A101 of Doxiadis et al. (1998) & AF052080 \\
\hline RhM-B1 & $\mathrm{B}$ & 571 & Rh*B101 of Doxiadis et al. (1998) & AF052084 \\
\hline RhM-B2 & $\mathrm{B}$ & 571 & Rh*B102 of Doxiadis et al. (1998) & AF052085 \\
\hline RhM-B3 & $\mathrm{B}$ & 571 & Rh*B103 of Doxiadis et al. (1998) & AF052086 \\
\hline RhM-O1 & $\mathrm{O}$ & 700 & Rh*O of Kermarrec et al. (1999) & AF094695 \\
\hline $\mathrm{RhM}^{-O} 2^{\mathrm{c}}$ & $\mathrm{O}$ & 700 & MamuO of Doxiadis et al. (1998) & AF094693 \\
\hline $\mathrm{RhM}-\mathrm{O} 2^{\mathrm{c}}$ & $\mathrm{O}$ & 868 & $\mathrm{Rh}^{*} \mathrm{O} * 01$ of Kermarrec et al. (1999) & AF071830 \\
\hline \multicolumn{5}{|l|}{ Yellow baboon } \\
\hline YeB-A & $\mathrm{A}$ & 571 & Baboon-1 of Kominato et al. (1992) & _- \\
\hline YeB-B & $\mathrm{B}$ & 571 & Baboon-2 of Kominato et al. (1992) & - \\
\hline \multicolumn{5}{|l|}{ Olive baboon } \\
\hline O1B-A & $\mathrm{A}$ & 690 & Baboon A of Diamand et al.(1997) & AF019416 \\
\hline O1B-B & $\mathrm{B}$ & 690 & Baboon B of Diamand et al.(1997) & AF019417 \\
\hline O1B-O & $\mathrm{O}$ & 690 & Baboon O of Diamand et al.(1997) & AF019418 \\
\hline
\end{tabular}

a $1211 \mathrm{bp}$ intron 6 is included.

${ }^{\mathrm{b}} 1156 \mathrm{bp}$ intron 6 is included.

${ }^{c}$ Doxiadis et al. (1998) and Kermarrec et al. (1999) reported rhesus macaque O allele sequences, but they are identical for the overlapped region. Therefore they were considered as one allele. 
from ThermoSequenase Kit (PE Biosystems).

Samples were electrophoresed using the ABI PRISM 377 sequencer (PE Biosystems). All the three PCR primers were used for sequencing. In addition, two other primers were used. They are: SN-04, an exon 7 upstream, 5'-ACGTCCTGCCAGCGCTTGTA-3', and SN-14, an intron 6, 5'-CCACCAGCACCCCCTCTTACT-3'.

CLASTAL W ver.1.6 (Thompson et al., 1994) was used for multiple alignment and phylogenetic tree construction. Phylogenetic trees were constructed by using the neighbor-joining method (Saitou and Nei, 1987). Phylogenetic networks (Bandelt, 1994; Saitou and Yamamoto, 1997) were also constructed.

\section{RESULTS AND DISCUSSION}

PCR amplification using primer combinations SN-10/ $\mathrm{SN}-02$ was conducted for Japanese macaque genomic DNA as template, and yielded about $0.5 \mathrm{~kb}$ fragments. We first compared those five sequences with the 17 published sequence data of rhesus macaque, crab-eating macaque, and olive baboon (Kominato et al., 1992; Diamand et al.,
1997; Doxiadis et al., 1998; Kermarrec et al., 1999). Sequences used in this study are listed in Table 1, and variant nucleotide sites are shown in Table 2 . There was no gap in the multiple aligned sequences. We did not find any heterozygotes in $487 \mathrm{bp}$ region of exon 7 for 5 individuals nor in the $1.0 \mathrm{~kb}$ region intron 6 for 2 individuals. The nucleotide diversity within Japanese macaque is $1.59 \%$ in mtDNA (Hayasaka et al. 1996), and the average heterozygosity estimated from protein polymorphism was $2.8 \%$ (Nozawa et al. 1996). This low diversity of Japanese macaque is compatible with our result.

We first constructed a phylogenetic tree for these 22 exon 7 sequences (Fig. 1). Most of branch lengths are very short, and bootstrap values are small. This tree is essentially unrooted, but we located the putative root in the branch separating A alleles and B alleles.

There are two hypotheses which can explain the sharing of blood group A and B antigens among primates. The first hypothesis posits that the sharing is the result of polymorphism being carried over from ancestral to descendent species in the course of primate evolution (the trans-species polymorphism hypothesis). The second

Table 2. Variant sites of ABO blood group gene in Old World monkeys

\begin{tabular}{|c|c|c|}
\hline & intron 6 & exon 7 \\
\hline $\begin{array}{l}\text { Site } \\
\text { number }\end{array}$ & $\begin{array}{l}136888 \\
376227 \\
954170\end{array}$ & $\begin{array}{l}1111111111111111111111111111 \\
2223333333444555555666666777 \\
6890111245479235599245679036 \\
7570789727068811837730002802\end{array}$ \\
\hline Consensus & $* * * * * *$ & $*$ GCCCCGGGGGCCGCCCGCGACGCGCG* \\
\hline JaM_B1 & $* * * * * *$ & 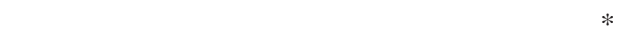 \\
\hline JaM_B2 & $* * * * * *$ & 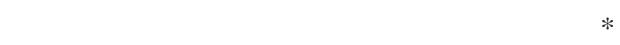 \\
\hline JaM_B3 & $* * * * * *$ & $c \cdots \cdots \cdots \cdots \cdot \cdots \cdot \cdots$ \\
\hline JaM_B4 & CATCCA & 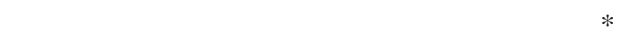 \\
\hline JaM_B5 & T GCT G G & Т................. \\
\hline CeM_A1 & $* * * * * *$ & 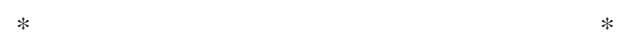 \\
\hline CeM_O & $* * * * * *$ & $* * \cdots \mathrm{T} \cdots \cdots \mathrm{T}$ T $\cdots \mathrm{T} \cdots \mathrm{TG} \cdots \cdots \mathrm{C}$ \\
\hline CeM_A2 & $* * * * * *$ & $* \ldots$ T. . A A T TAG $\cdot$ T $\cdots$ TG $\cdots$. . * \\
\hline CeM_A3 & $* * * * * *$ & $* \cdots$ T. $\cdots$ AAATT $\cdots$ T $\cdots$ TG $\cdots$ C \\
\hline CeM_B1 & $* * * * * *$ & $* \ldots \mathrm{A}_{\mathrm{A}} \ldots \mathrm{A} \cdot \ldots \mathrm{A} \cdot \mathrm{T}$ \\
\hline CeM_B2 & $* * * * * *$ & $* \ldots \ldots \ldots \ldots \ldots \ldots \ldots \ldots \ldots \ldots$ \\
\hline RhM_A1 & $* * * * * *$ & $* \cdots$ T $\cdots$. T TAG $\cdots$. TG $\cdots$ A $\cdot$ C \\
\hline RhM_O1 & $* * * * * *$ & $* \cdots$ T. . . A T TAG $\cdot$ T $\cdots$ TG $\cdots$ C \\
\hline RhM_O2 & $* * * * * *$ & $* \ldots \ldots \ldots \ldots \ldots \ldots \ldots \ldots$ \\
\hline RhM_B1 & $* * * * * *$ & $* \ldots \ldots \ldots \ldots \ldots \ldots \ldots \ldots$ T $\ldots \ldots$ \\
\hline RhM_B2 & $* * * * * *$ & $* \ldots$ A $\cdots \ldots \ldots \ldots \ldots \ldots$ A \\
\hline RhM_B3 & $* * * * * *$ & $* \ldots \ldots \ldots \ldots \ldots \ldots \ldots$ \\
\hline YeB_A & $* * * * * *$ & $* \mathrm{C} \cdots \mathrm{T} \cdots \cdots \mathrm{AG} \cdots \cdots \mathrm{TGA} \cdots * *$ \\
\hline YeB_B & $* * * * * *$ & $* \mathrm{C} \cdots \mathrm{T} \cdots \ldots \cdot \mathrm{AG} \cdot \mathrm{T} \cdots \ldots *$ \\
\hline OIB_A & $* * * * * *$ & $* \mathrm{C} \cdots \mathrm{T} \cdots \cdots \mathrm{AG} \cdots \cdots \mathrm{TGA} \cdots \mathrm{C}$ \\
\hline OIB_B & $* * * * * *$ & $* \mathrm{c} \cdots \mathrm{T} \cdots \cdots \mathrm{AG} \cdots \cdots \cdots \mathrm{C}$ \\
\hline OIB_O & $* * * * * *$ & $* \mathrm{C} \cdots \mathrm{T} \cdot \mathrm{A} \cdots \mathrm{TTAGGT} \cdots \mathrm{TGA} \cdots \mathrm{C}$ \\
\hline
\end{tabular}

Notes. The variant sites in intron 6 to exon 7 are shown. Dots and asterisks denote identical nucleotides with the consence and nonexamined positions, respectively. In this region, there are no gaps within Old World monkey sequences. Sites 1643 and 1650 are the critecal sites between A and B allele. All Japanese macaque sequences have the same nucleotides as other primate B type alleles in these two critical setes. 


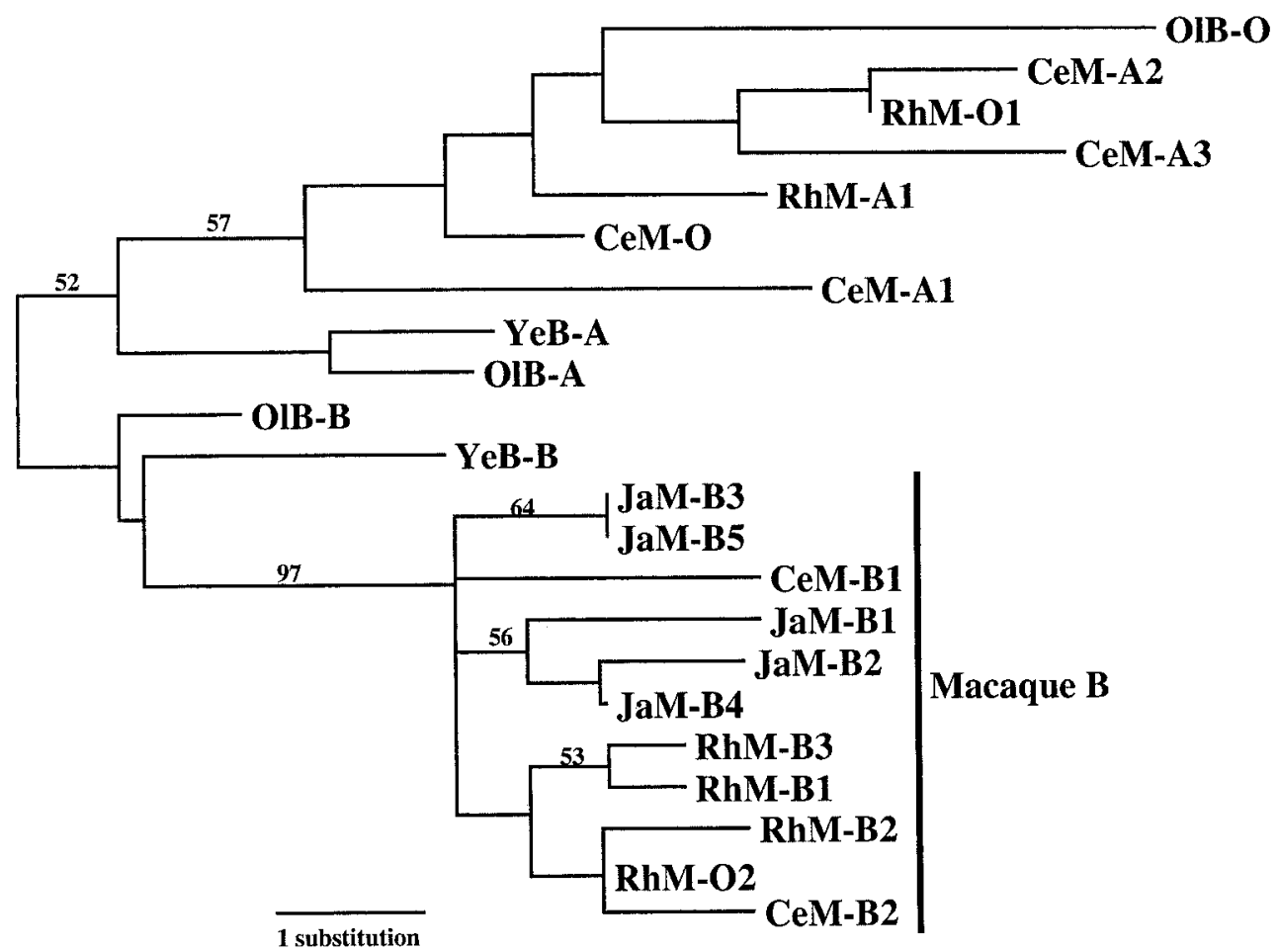

Fig. 1. The phylogenetic tree of the ABO blood group gene exon 7 in Old World monkeys. This tree is rooted in the branch between Atype and B-type sequences. Bootstrap values only greater than $50 \%$ are shown.

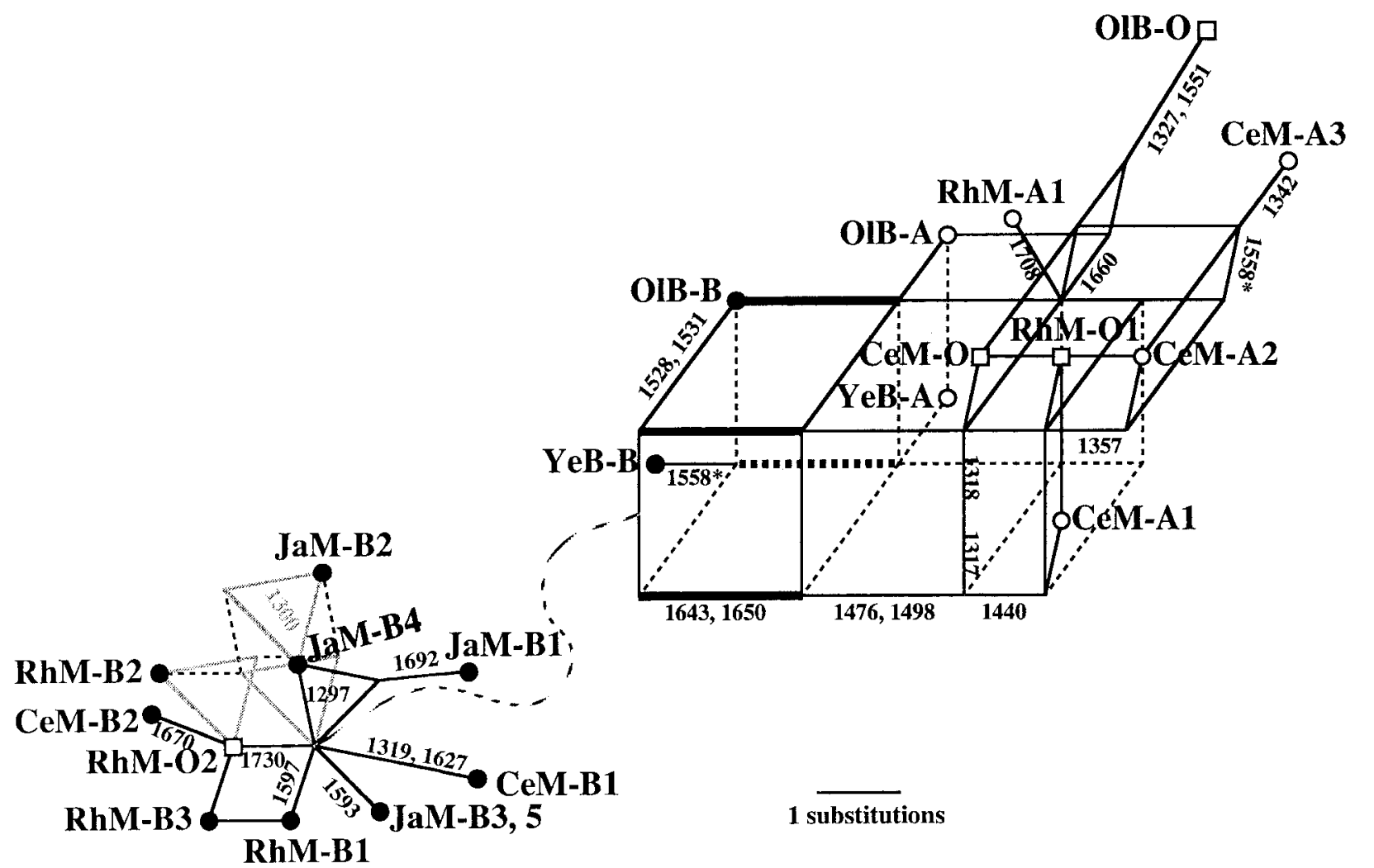

Fig. 2. The phylogenetic network of the ABO blood group gene in Old World monkeys. The numbers on edges show site numbers. The thick-lined edges are the two critical sites between A and B alleles. Nodes with open circles, full circles, and squares denote A type, B type, and $\mathrm{O}$ type alleles, respectively. A curved broken line denotes connection between macaque B type alleles and others (no substitution). Substitutions that were thought to occurred parallel is shown with asterisk. 
invokes parallel evolution as an explanation for the interspecific sharing of antigenic determinants. The former hypothesis was supported by Martinko et al. (1993); the latter was proposed by Saitou and Yamamoto (1997) and is more consistent with partial intron 6 hominoid sequence data described by O'hUigin et al. (1997). In Old World monkey, we should consider these two hypotheses. In Fig. 1, the bootstrap value for the branch between A allele and $\mathrm{B}$ allele group is $46 \%$. We therefore can't determine which hypothesis is correct. The bootstrap value for the branch between macaques B alleles and other alleles is high, 97\%. Therefore, the polymorphism of A and B alleles seems to be maintained at least before the speciation of three macaques.

We then constructed the phylogenetic network of those $\mathrm{ABO}$ exon 7 sequences for three macaque species and two baboon species (Fig. 2). Sequence data between site 1297 and site 1730 were used. Numbers on edges show site numbers, and the thick-lined branches denote the two critical sites between A and B alleles. Sequences shown with open circles are A type alleles, and those shown with full circles are B type. All $O$ type alleles shown with open squares are located within the A type allele variations except for one $\mathrm{O}$ type allele (RhM-O2), which is within the $\mathrm{B}$ type group. There are many incompatibilities, and the resulting network is complex. All the incompatibilities are presented in the form of reticulation in this network except for site 1558. The sequences YeB-B, OlB-O, CeMO, RhM-O1, CeM-A2, and CeM-A3 share the same nucleotide $\mathrm{T}$ at this site (other sequences are $\mathrm{C}$ ). But YeB-B is separated from other 1558-T group sequences with more than 8 substitutions, so we inferred that the substitutions from $\mathrm{C}$ to $\mathrm{T}$ in 1558 occurred twice in the YeB-B lineage and in the lineage leading to OlB-O, CeM-O, RhM-O1, CeM-A2, and CeM-A3, designated with asterisks.
In this network, the B type alleles of macaque and RhM$\mathrm{O} 2$ formed a clear cluster, as in the NJ tree in Fig. 1 (the bootstrap value is $97 \%$ ), and the topology within the B type alleles are similar to that of the NJ tree. The CeM-A2, RhM-O1, and CeM-O share the same nucleotide at site 1558 , and their distances are small (one or two substitutions), but CeM-O is located outside of the cluster that contains CeM-A2, RhM-O1, CeM-A3, OlB-O, and RhM-A1 in the NJ tree, and the distance between CeM-O and RhMO1/CeM-A2 in the NJ tree is bigger than that of the network. This discrepancy between the tree and the network may be caused from incompatibilities among sites 1357-1440, sites 1528-1531, and site 1558.

Based on the the phylogenetic network shown in Fig. 2, the B type alleles of three macaques (Japanese macaque, rhesus macaque, and crab-eating macaque) are closely related to each other, compared to the A type alleles of rhesus and crab-eating macaques. It is thus clearly shown that the A and B polymorphism arose before the speciation of these macaques. The nucleotide differences between A type and B type alleles in macaque are large (at least 4 including the critical sites), while baboon alleles are located within the macaque variation. There is one baboon specific site at 1285 , and the nucleotide is $\mathrm{C}$ in baboons, while it is $\mathrm{T}$ or $\mathrm{G}$ in macaques (see Table 2). Furthermore in intron 6, there are some species specific sites for baboon and macaque (Noda et al., unpublished data). These suggest that baboon alleles constitute a monophyletic group.

From the network and these informations, we chose one tree (Fig. 3). This tree has two major differences from that of Fig. 1. First one is the location of baboon sequences. The baboon sequences are monophyletic in Fig. 3 , while they are scattered and are not monophyletic in the tree of Fig. 1, especially OlB-O is separated from other

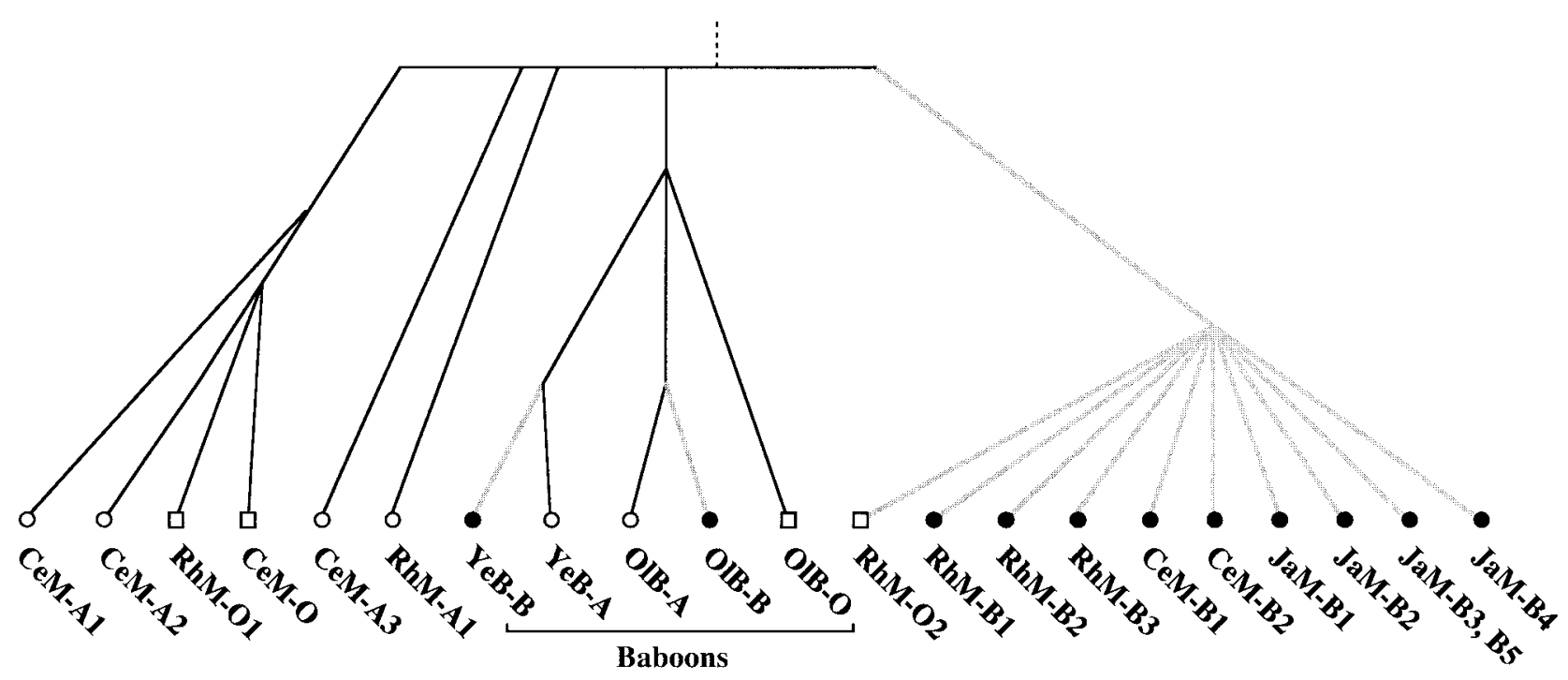

Fig. 3. A possible rooted tree of the Old World monkey ABO blood group gene based on the phylogenetic network of Fig. 2. 
baboon sequences. The second one is the location of A-like sequences. For example, RhM-A1 and CeM-A3 are located outside of the other A-like sequences in Fig. 3, but they locate inside of the A-like sequence cluster in Fig. 1.

In Fig. 4, we show the hypotheses on the evolutionary history of the ABO blood group gene in macaque and baboon. Fist two figures show the hypotheses which have already been proposed; (A) convergent evolution (B type allele evolved independently in each macaque and baboon lineage after speciation) and (B) ancestral polymorphism.
Under the hypothesis (A), the parallel substitutions were supposed to occur in two critical sites (shown as thick line) in Fig. 2, and the parallel substitutions were supposed to occurr in sites that are incompatible with critical sites (for example, site numbers 1357-1440 and 1528-1531) under hypothesis (B). We must assume that parallel changes occurred many times under both hypotheses. Fig. 4 (C) shows our hypothesis that is a mixture of convergent evolution and ancestral polymorphism. This is based on the tree of Fig. 3, and the number of substitu-
(A)

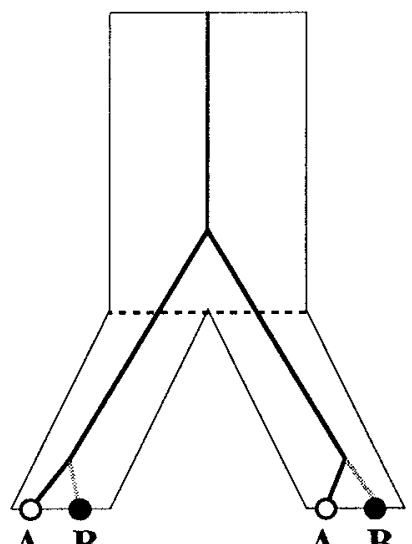

A B

Macaque
Baboon
(B)

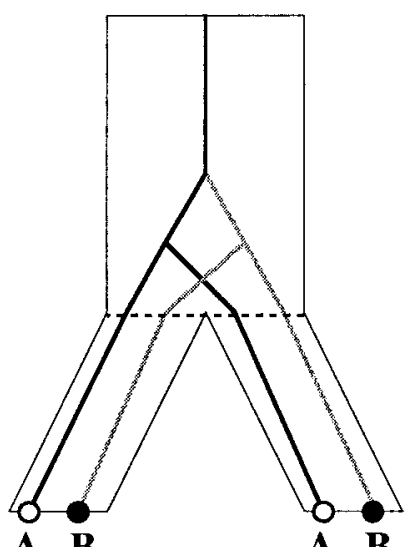

A B

\section{Macaque}

(C)

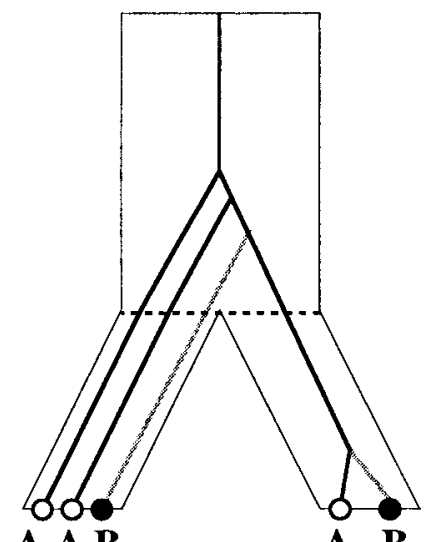

A A B

Macaque

A B

Baboon

(A) convergent evolution, (B) ancestral polymorphism, and (C) the hypothesis based on the tree of Fig. 3 . The dotted line shows the speciation of macaque and baboon.

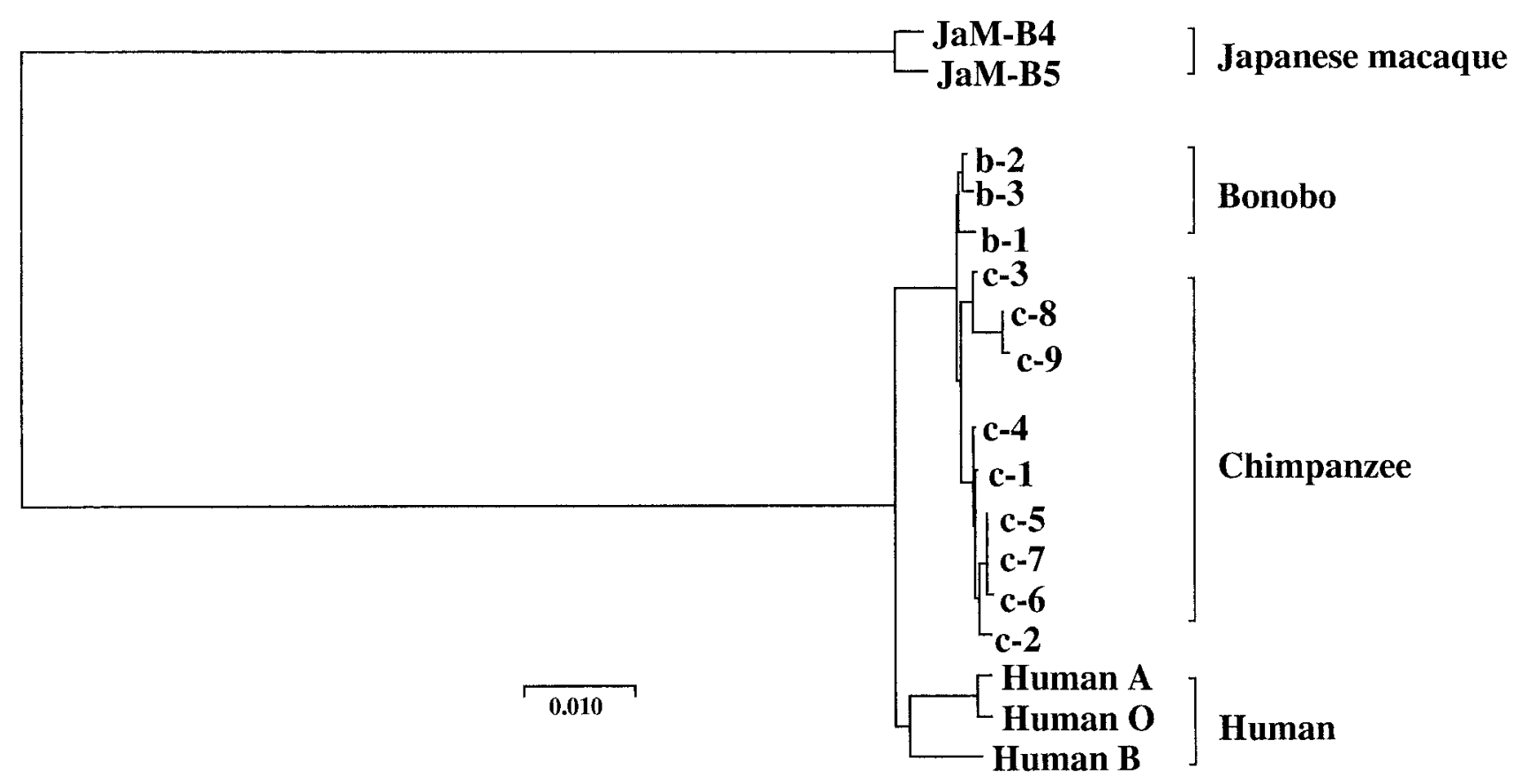

Fig. 5. The phylogenetic tree of the ABO blood group gene intron 6 and exon 7 in Japanese macaque, chimpanzee, bonobo, and human. 
tions in this scheme is less than those of (A) and (B). Thus, this tree looks most plausible.

We also aligned two Japanese macaque sequences spanning exon 6 to exon 7 with chimpanzee and bonobo sequences spanning intron 6 and partial exon 7 (Kitano et al., 2000) as well as human (Olsson and Chester, 1998), and constructed a phylogenetic tree (Fig. 5). In this tree, three genera (Homo, Pan, and Macaca) clearly make their own clusters. The distance between hominoids and macaque is substantial, thus the convergent evolution hypothesis is more compatible with this tree. The difference between chimpanzees and Japanese macaque is around $16-17 \%$. If this corresponds to $25-30 \mathrm{MY}$ divergence between hominoid and Old World monkeys, coalescent of the two Japanese macaque sequences $(0.5 \%)$ is estimated to be 0.7-0.9 MYA under the assumption of molecular clock. The nucleotide diversity of Japanese macaque was estimated to be about $1.5 \%$ in the mitochondrial DNA, and the divergence times among Japanese macaques were estimated to be 0.19-0.37 MYA (Hayasaka et al., 1996). It should be noted that the effective population size of nuclear DNA is four times larger than that of mitochondrial DNA. Observed difference of coalescent times between the $\mathrm{ABO}$ gene and mitochondrial DNA is more or less compatible with this expected difference of the effective population size.

In conclusion, the type change between A and B alleles of the $\mathrm{ABO}$ blood group gene occurred independently at least two times in Old World monkey, in the macaque/baboon ancestor and the baboon lineages.

We thank Dr. Kenta Sumiyama for various discussions in the earlier version. We also thank Dr. Antoine Blancher for informing us the paper by Bernard and Ruffié (1972). This study was partially supported by grants in aid for scientific studies from Ministry of Education, Science, Sports and Culture, Japan to NS.

\section{REFERENCES}

Bandelt, H. J. (1994) Phylogenetic networks. Verh. Naturewiss. Ver. Hamburg (NF) 34, 51-71.

Bernard, J. and Ruffié, J. (1972) Hématologie Géographique, Tome II. Mason et Cie, Paris (in French).

Blancher, A. and Socha, W. W. (1997) The ABO, Hh, and Lewis blood group in humans and nonhuman primates. In: Molecular biology and evolution of blood group and MHC antigens in primates (eds.: A. Blancher, J. Klein, and W. W. Socha), pp. 31-92. Springer-Verlag Berlin Heidelberg New York.

Crow, J. F. (1993) Felix Bernstein and the first human marker locus. Genetics 133, 4-7.
Diamand, D. C., Fagoaga, O. R., Nehlsen-Cannarella, S. L., Bailey L. L., and Szalay, A. (1997) Sequence comparison of baboon $\mathrm{ABO}$ histo-blood group alleles: lesions found in $\mathrm{O}$ alleles differ between human and baboon. Blood Cells Mol. Dis. 23, 242-251.

Doxiadis, G. G. M., Otting, N., Antunes, S. G. M., de Groot, N. G., Harvey, M., Doxiadis, I. I. N., Jonker, M., and Bontrop, R. E. (1998) Characterization of the ABO blood group genes in macaques: evidence for convergent evolution. Tissue Antigens 51, 321-326.

Hayasaka, K., Fujii, K., and Horai, S. (1996) Molecular phylogeny of macaques: implications of nucleotide sequences from an 896-base pair region of mitochondrial DNA. Mol. Biol. Evol. 13, 1044-1053.

Kermarrec, N., Roubinet, F. Apoil, P.-A., and Blancher, A. (1999) Comparison of allele $\mathrm{O}$ sequences of the human and non-human primate $\mathrm{ABO}$ system. Immunogenetics 49, 517-526.

Kitano, T., Noda, R., Sumiyama, K., Ferrell, R. E., and Saitou, N. (2000) Gene diversity of chimpanzee ABO blood group genes elucidated from intron 6 sequences. J. Heredity (in press).

Kominato, Y., McNeill, P. D., Yamamoto, M. Russell, M., Hakomori, S., and Yamamoto, F. (1992) Animal histo-blood group ABO genes. Biochem. Biophys. Res. Com. 189, 154164.

Martinko, J. M., Vincek, V., Klein, D., and Klein, J. (1993) Primate ABO glycosyltransferases: evidence for trans-species evolution. Immunogenetics 37, 274-278.

Nozawa, K., Shotake, T., Minezawa M., Kawamoto, Y., Hayasaka, K., and Kawamoto, S. (1996) Population-genetics studies of the Japanese Macaque, Macaca fuscata. In: Variations in the Asian macaques. (eds: T. Shotake and K. Wada), pp 1-37. Tokai University Press, Tokyo

O'hUigin, C., Sato, A. and Klein, J. (1997) Evidence for convergent evolution of $\mathrm{A}$ and $\mathrm{B}$ blood group antigens in primates. Hum. Genet. 101, 141-148.

Olsson, M. L. and Chester, M. A. (1998) Heterogeneity of the blood group Ax allele: genetic recombination of common alleles can result in the Ax phenotype. Transfus. Med. 8, 231-238.

Saitou, N., and Nei, M. (1987) The neighbor-joining method: a new method for constructing phylogenetic trees. Mol. Biol. Evol. 4, 406-425.

Saitou, N., and Yamamoto, F. (1997) Evolution of primate ABO blood group genes and their homologous genes. Mol. Biol. Evol. 14, 399-411.

Thompson, J. D., Higgins, D. G. and Gibson, T. J. (1994) CLASTAL $\mathrm{W}$ : improving the sensitivity of progressive multiple sequence alignment through sequence weighting, position-specific gap penalties and weight matrix choice. Nucleic Acids Res. 22, 4673-4680.

Yamamoto, F. (1995) Molecular genetics of the ABO histo-blood group A System. Vox Sang. 69, 1-7.

Yamamoto, F., Clausen, H., White, T., Marken, J. and Hakomori, S. (1990) Molecular genetic basis of the histo-blood group ABO system. Nature 345, 229-233.

Yamamoto, F., McNeill, P. D., and Hakomori, S. (1995) Genomic organization of histo-blood group ABO genes. Glycobiology 5, 51-58. 